\title{
Regression Tests to Expose Change Interaction Errors
}

\author{
Marcel Böhme \\ Bruno C.d.S. Oliveira \\ Abhik Roychoudhury \\ National University of Singapore \\ \{marcel, oliveira, abhik\}@comp.nus.edu.sg
}

\begin{abstract}
Changes often introduce program errors, and hence recent software testing literature has focused on generating tests which stress changes. In this paper, we argue that changes cannot be treated as isolated program artifacts which are stressed via testing. Instead, it is the complex dependency across multiple changes which introduce subtle errors. Furthermore, the complex dependence structures, that need to be exercised to expose such errors, ensure that they remain undiscovered even in well tested and deployed software. We motivate our work based on a well tested and stable project - GNU Coreutils - where we found that one third of the regressions take more than two (2) years to be fixed, and that two thirds of such long-standing regressions are introduced due to change interactions for the utilities we investigated.

To combat change interaction errors, we first define a notion of change interaction where several program changes are found to affect the result of a program statement via program dependencies. Based on this notion, we propose a change sequence graph (CSG) to summarize the control-flow and dependencies across changes. The CSG is then used as a guide during program path exploration via symbolic execution - thereby efficiently producing test cases which witness change interaction errors. Our experimental infrastructure was deployed on various utilities of GNU Coreutils, which have been distributed with Linux for almost twenty years. Apart from finding five (5) previously unknown errors in the utilities, we found that only one in five generated test cases exercises a sequence that is critical to exposing a change-interaction error, while being an order of magnitude more likely to expose an error. On the other hand, stressing changes in isolation only exposed half of the change interaction errors. These results demonstrate the importance and difficulty of change dependence-aware regression testing.
\end{abstract}

Categories and Subject Descriptors: D.2.5 [Software Engineering]: Testing and Debugging

General Terms: Theory, Algorithms, Experimentation

Keywords: Test Generation, Graph-based Search

Permission to make digital or hard copies of all or part of this work for personal or classroom use is granted without fee provided that copies are not made or distributed for profit or commercial advantage and that copies bear this notice and the full citation on the first page. Copyrights for components of this work owned by others than ACM on the first page. Copyrights for components of this work owned by others than ACM
must be honored. Abstracting with credit is permitted. To copy otherwise, or republish, to post on servers or to redistribute to lists, requires prior specific permission and/or a fee. Request permissions from Permissions@acm.org.

ESEC/FSE'13, August 18-26, 2013, Saint Petersburg, Russia Copyright 2013 ACM 978-1-4503-2237-9/13/08 ...\$15.00 http://dx.doi.org/10.1145/2491411.2491430

\section{INTRODUCTION}

Changes even to well-tested software projects can introduce subtle bugs of varying severity that may be exposed only years later. Such change-based errors in deployed software come in two forms. First of all, bug fixes may introduce new bugs. For instance, $\mathrm{Gu}$ et al. [7] mention that feature additions or bug fixes, introduce new bugs in $9 \%$ of cases. Secondly, a subtle or poorly understood "interaction" among various changes may introduce hard-to-find errors in well-tested code, which then get deployed. In this paper, we focus on test generation to expose such subtle change interaction errors (CIEs).

Subtle change interaction errors can be found in many well-tested and deployed software projects. In our study on GNU Coreutils, we found that every fifth bug fix actually patches regressions introduced in an earlier commit. About one third of these regressions take more than two (2) years to find and fix ${ }^{1}$, despite the tool set being rather well tested. Note that $\sim 21 \%$ of the total commits update the comprehensive test suite, while only $30 \%$ actually update the utilities (the remaining $49 \%$ are related to maintenance, like documentation, the build process, or ambiguous error messages). Thus, it is surprising that on utilities with such well-updated test-suites, errors due to change interaction will remain for two years. In fact, the GNU Coreutils have been dispatched with almost every Linux distribution for the last 20 years!! This led us to think that change interaction errors, which stress subtle dependencies across changes, may be hard-tofind due to most regression testing methods being focused on some form of coverage.

At this point, we step back and review the recent regression testing research which focus on program changes. A recent work [17] presents criteria and experiments for the interaction among program changes but does not suggest any method for integrating them into regression testing. Among the works achieving change-aware test generation, some study only independent program changes $[1,13]$. Several of the testing methods attempt to achieve either a structural coverage of changed statements or some other structural coverage (such as branch outcome coverage) in the modified program (e.g., see [22]). Since coverage-based methods may not stress the semantic effect of the changes, attempts have been made to take a powerful symbolic execution based path exploration engine, and adapt it to the presence of program changes. Since symbolic execution captures the semantic effect of program changes, the hope is that the semantic effect of a change can be propagated through

\footnotetext{
${ }^{1}$ In our experience, most bugs are fixed days after the report.
} 
such methods. On the other hand, since a full-fledged symbolic execution based path exploration can be exceedingly slow, these methods employ various pruning strategies to cull away program paths which cannot reach or propagate changes (e.g., see [18]). Other authors suggest to statically compute the program slices for every change and dynamically employ symbolic execution upon these slices to exercise all paths that are affected by a changed statement (e.g., see [12]). However, in all of these works, the set of changes in a program is treated in an aggregate fashion. The flows/dependencies across changes are not systematically explored/exploited for generating test cases.

In this paper, we present a test generation method to systematically explore and expose subtle errors arising due to the "interaction" among program changes. Since any such change interaction leading to errors is inherently dynamic, we first statically approximate the relationships among the changes. Our approximation is called change sequence graph (CSG) which captures (i) the control-flow across the changed statements and (ii) the control-flow to control locations at which multiple changes may interact, leading to unexpected semantic effects. These interaction locations are computed based on the program dependencies across multiple changed statements. The CSG is then used as a definitive guide to find out the sequence of control locations that need to be visited for exposing potential change interaction errors. These control locations are visited systematically by programming a graph-based search strategy on top of the directed symbolic execution engine, Otter [9].

Experimental results from our approach on Coreutils show the prevalence of change interaction errors among regression bugs. We note that the GNU Coreutils tool-set is a collection of Linux utilities which have been widely tested. In particular, every fifth commit to the repository updates a comprehensive test suite that exists for more than twenty years, and the tool set was further tested by the authors of klee [3] and test-zesti [10] (reporting 3 and 2 errors, respectively). Despite such extensive testing, we found and reported five verified, previously unknown regression errors, apart from many known errors. Among other notable findings, we noticed that two in three differential errors can be classified as change interaction errors. We also found that only half of the CIEs were exposed by a testing algorithm that target changes in isolation, but does not account for their interaction. This clearly demonstrates the importance of change interaction-aware regression testing.

In summary, the contributions of this paper are:

- Change Interaction Errors: We identify and formalize change interaction errors: errors that happen in evolving software, which arise due to the combined semantic impact of multiple changes. We argue for the importance of this class of errors with a study of regression on GNU coreutils over a period of 5 years.

- Detection Method for CIEs based on CSGs: We propose a datastructure called a change sequence graph to capture potential sequences of changed statements and interaction locations in an execution of a program. Using CSGs we show a detection method which stresses sequences in the graph to expose CIEs.

- Implementation and Empirical Evaluation: Our CIEs detection method has been implemented and an empirical evaluation using that implementation was conducted to evaluate its effectiveness.

\section{REGRESSION IN GNU COREUTILS}

To study software regression, we looked at the repository of GNU Coreutils, which has been actively developed and maintained for more than twenty years. Our results show that within the last five years every fifth bug fix actually patches regressions introduced earlier and that $30 \%$ of such regressions take more than 2 years to be fixed.

\subsection{Statistics of Regression}

It is possible to access the history of every change commited to the source code repository of GNU Coreutils since Oct' $92^{2}$. Usually, these commits are accompanied by a commit message that describes the relevance and intention of the change. The commits to the repository of GNU Coreutils are categorized as changes to particular tools, or as build, tests, maint [enance], amongst others. The developers adopted this commit message labeling about five years ago. This allows us to distinguish code-changing commits ${ }^{3}$ from maintenance commits. Parsing the commit messages for keywords, such as "bug", "fix", or "regression", we were able to approximate how many of the code-changing commits are bug fixes and feature additions. If the commit message contained "introduced" or "regression", we could derive whether a bug fix was actually patching regressions introduced earlier. Often, a regression-fixing commit would reference the regression-introducing commit. Thus, we can measure the time in-between. Note that this process may yield false negatives. While we manually validated the reported ones, we may miss some real regressions. As the commits have been nicely categorized in the last five years, we looked at those between Jan'08 and Feb'13. However, regression-fixing commits can reference regression-introducing commits that were submitted much earlier. Given the X- and the (logarithmic) $\mathrm{Y}$-axis in Figure 1, the graph shows that $\mathrm{X}$ percent of the regression-introducing commits 1 ) require more than $Y$ days to be found and fixed (solid line), and 2) contain more than $Y$ Changed Lines of Code (CLoC; dashed line).

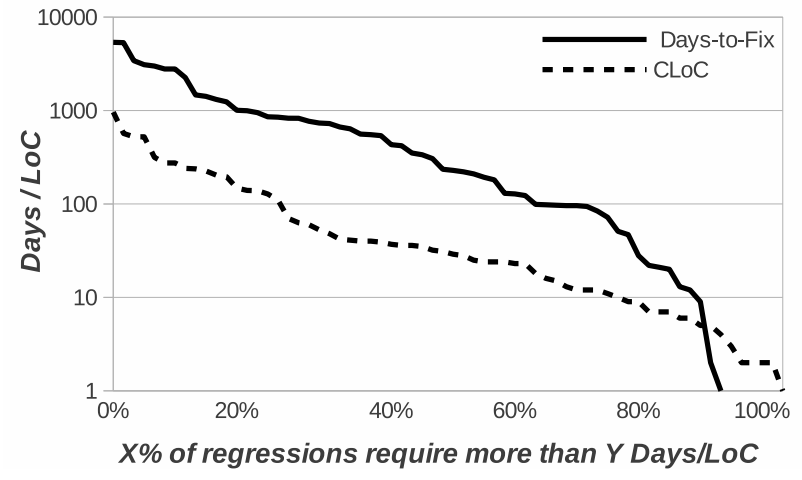

Figure 1: Regression Statistics - GNU Coreutils

Results. About $30 \%$ of the $2.6 \mathrm{k}$ commits in the recent 5 years are code changes - feature additions and bug fixes in roughly equal shares. Interestingly, every fifth bug fix actually patches regressions introduced in an earlier commit. The following observations are further corroborated by package maintainer, Pádraig Brady, via email-exchange:

${ }^{2}$ http://git.savannah.gnu.org/cgit/coreutils.git

${ }^{3}$ Code-changing commits are labeled by the changed tool. 
O.i $30 \%$ of the regressions introduced in earlier commits take more than 2 years to find and fix despite a comprehensive and well-maintained test suite $(\sim 21 \%$ of the total commits update the test suite)

O.ii $45 \%$ of the regressions are introduced when more than 35 LoC are changed (while only about $25 \%$ of the codechanging commits modify 35 LoC or more).

This led us to suspect that the changed behaviour introduced by the syntactic changes to the tools is not properly tested. In particular, we consider the subtle interplay of many code changes as reasons for regressions to be exposed so late. We call this type of errors change interaction errors. Indeed, as discussed in Section 7, we find that $66 \%$ of the errors introduced in earlier commits can only be exposed by input exercising certain critical sequences of changed statements. It turns out that only one in five tests inputs exercise a critical sequence, while such test cases are 15 times more likely to expose an error. In the remainder of this section, we have a closer look at one of the regression errors.

\subsection{Buffer Overflow in cut}

During our investigations, we found and reported a buffer overflow in the tool cut of GNU Coreutils which was introduced as a regression error in commit ec48bead and manifests as SEG_FAULT for the failing test input. ${ }^{4}$ Buffer overflows can be exploited maliciously to gain root access to affected computers [4]. This issue is particularly critical for systems that are dispatched with almost every Linux distribution, such as GNU Coreutils, which contains wellknown command-line tools, such as cp, mv, rm, echo, and cut. Fortunately, in the five years preceeding this paper the package maintainer of GNU Coreutils had to fix only 10 SEG_FAULTs. ${ }^{5}$ However, a surprising 6 out of 10 are regression errors introduced in earlier commits.

\subsubsection{The Anatomy of a Regression}

In simple terms, the tool cut takes a set of number ranges, a file, and an optional output-delimiter as input and prints the content of every line in the specified file within the specified ranges, optionally separated by the specified outputdelimiter. For instance, the command in Figure 2, uses "hello world" as input to the cut utility - which prints the range between the 2 nd and $3 \mathrm{rd}$ character, and from the 7 th character onwards, both ranges separated by "," (comma).

\$ echo "hello world" I cut -output-del=, -b2-3,7-
el, world

Figure 2: Linux Terminal - the output of cut

Problem. If there are no finite ranges (e.g., 7-), then too much memory is unnecessarily allocated.

Specifically, if max_range_endpoint is set in line 504 of Figure 3 or earlier, then the array printable_field is allocated max_range_endpoint of memory (line 509). If output_delimiter_specified, then printable_field is unnecessarily (but successfully) accessed at eol_range_start in line 266. Note, if eol_range_start>max_range_endpoint, then max_range_endpoint is set to eol_range_start in line 504.

${ }^{4}$ Report and fix avail. at http://debbugs.gnu.org/13627.

${ }^{5}$ Again, we analysed commit messages in the repository. Due to false negatives the actual number may be greater.
Intended Change. Memory allocation only if necessary. Specifically, only if max_range_endpoint is set, allocate the array printable_field with max_range_endpoint of memory. Only if output_delimiter_specified and max_range_endpoint is set, then the array printable_field shall be accessible in line 534 .

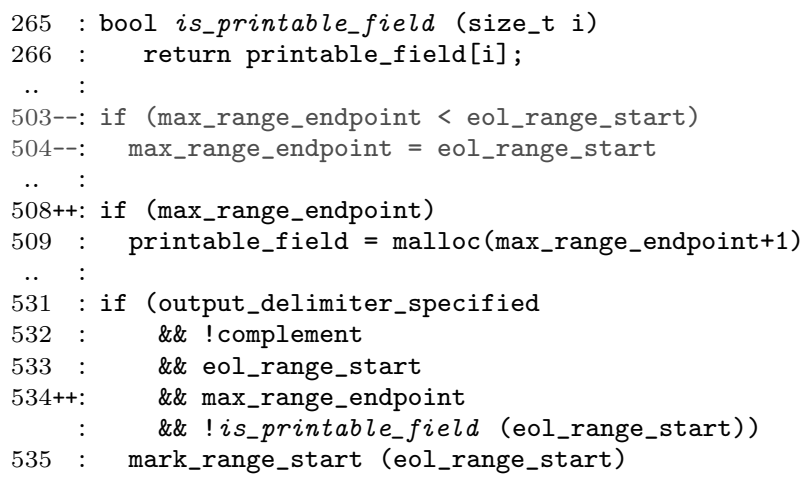

Figure 3: SEG_FAULT introduced in cut

Actual Changes. The developer applies three code changes. Every change is essential to fix the memory leak.

Specifically, the developer C.1) adds that printable_field is allocated only if max_range_endpoint is set (line 508), C.2) adds that printable_field is accessed only if max_range_endpoint is set (line 534), and C.3) removes that max_range_endpoint is set to eol_range_start if eol_range_start > max_range_endpoint (lines 503-504). Note, all changes are essential to fix the memory leak. For instance, without change C.3, the variable guarding the memory allocation is always set, rendering the additional checks of changes $C .1$ and C.2 redundant.

Regression Error. If finite ranges are specified, then unallocated memory can be accessed, yielding a SEG_FAULT. Specifically, if 1) max_range_endpoint is set, 2) max_range_endpoint < eol_range_start, and 3) output_delimiter_specified is set, then the array printable_field is accessed out-of-bounds at eol_range_start in line 266.

\subsubsection{Combined Semantic Impact of Changes}

The observation of the regression error depends on the execution of both changes, C.1 and C.2. They have a combined "semantic impact" on the same program location the memory access. Specifically, the allocation of memory for printable_field in line 509 depends on the code added with change $C .1$. The access of memory in printable_field in line 266 depends on the code added with change C.2. Because the success of accessing an array also depends on the memory allocation for this array, both changes have a combined impact at the memory access location. So, the memory access at line 266 is called interaction location of C.1 and C.2. The sequences in which the changes can be executed are depicted in Figure $4 .{ }^{6}$ Note, C.3 is not part of the presented graphs since a deletion does not manifest in the changed version $P^{\prime}$.

${ }^{6}$ For brevity, we removed sequences that contain a change but no memory allocation or access. 


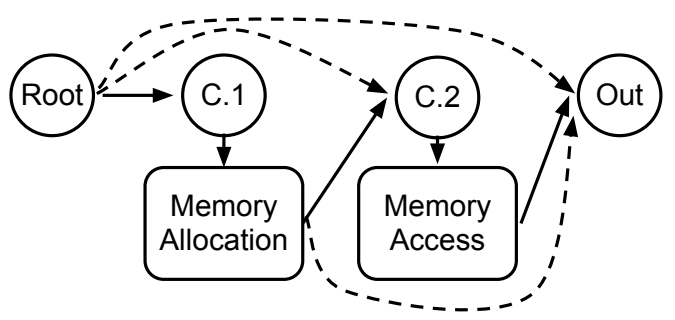

Figure 4: Input can exercise these change sequences.

It is insufficient to test both changes in isolation. The regression error is only observable for (some) input that exercises both changes - the sequence following the solid lines in Figure 4. The buffer overflow is not observable for input exercising only change $C .1$ but not $C .2$ and neither for input exercising only change $C .2$ but not $C .1$ - sequences exercising one of the dashed lines in Figure 4. Hence, we call this regression a change interaction error.

\section{ERRORS IN SOFTWARE EVOLUTION}

This section formally describes classes of errors that can occur during software evolution. In particular, we are interested in a class of errors, arising from the interaction of multiple changes, that we call change interaction errors (CIE). To establish the context of CIEs, we also define a useful generalization of regression errors, which we call differential errors.

\subsection{Preliminaries}

For the definitions in this section, we will assume two successive versions of a program, $P$ and $P^{\prime}$, and an oracle $S$. The oracle $S$ specifies the intended behavior for $P$ and $P^{\prime}$. As such, it is expected that for all input $i$ executed on $P^{\prime}$, the output is observationally equivalent ${ }^{7}$ to executing $i$ on the oracle $S$. Note that we abstract over what the oracle $S$ is: it could be a specification, the ultimate final version of a program, a validating test suite, or some other artifact that could be used to validate expected behaviour. Using earlier version $P$, the changed version $P^{\prime}$ and intended behavior $S$ of $P$ and $P^{\prime}$, we can more formally define what we mean by a regression error.

\section{Definition 1 (Regression Error)}

A regression error is observed on input $i$ if and only if $P(i) \not \equiv P^{\prime}(i)$ and $P(i) \equiv S(i)$.

In other words, a regression error happens when for some input $i$ the earlier version, $P$, works as expected but the new version, $P^{\prime}$, does not work anymore. Note that this definition does not prevent $P^{\prime}$ from exposing the correct behaviour for some other input, which fails in $P$ w.r.t. $S$. Therefore, our definition of regression error captures the common situation in which the initial version $P$ may have some errors that are intended to be fixed in $P^{\prime}$, but while $P^{\prime}$ is fixed for some inputs, it starts behaving incorrectly for some other inputs. An intended software quality improvement turns into a possible detoriation of the software quality.

\footnotetext{
${ }^{7}$ Two programs $P$ and $P^{\prime}$ are observationally equivalent for an input $i, P(i) \equiv P^{\prime}(i)$, if the relevant program output produced by executing $i$ on $P$ and $P^{\prime}$ is the same.
}

\subsection{Differential Errors}

In the context of software evolution we often find the need for a notion more general than that of a regression error. We call this notion differential error.

\section{Definition 2 (Differential Error) \\ A differential error is observed on input $i$ if and only if $P(i) \not \equiv P^{\prime}(i)$ and $P^{\prime}(i) \not \equiv S(i)$.}

In other words, a differential error happens when, for some input $i$, the changed version $P^{\prime}$ works differently from both, the earlier version $P$ and the intended behavior $S$. There are two interesting situations. The situation in which the earlier version $P(i)$ worked as expected $(P(i) \equiv S(i))$ is just equivalent to the definition of regression error. On the other hand, the situation in which the earlier version $P(i)$ did not work as expected either $(P(i) \not \equiv S(i))$ cannot be called regression error. So we call it differential error. This captures a situation, e.g., of an incomplete fix. The developer intends to fix the behaviour of $P$, when test cases $i$ and $j$ failed on $P$ w.r.t. $S$. But while $i$ may now pass in the fixed version $P^{\prime}$ and $j$ produces different output, $j$ may still fail on $P^{\prime}$ w.r.t. $S-$ the fix was incomplete. In practice, it is helpful to characterize situations in which several intermediate "fixes" are implemented until an ultimate version meets the expectations.

\subsection{Change Interaction Errors}

A change interaction error is a special kind of differential error. Informally, a change interaction error happens when multiple changes are introduced in a program, and those multiple changes interact in unexpected ways. More formally we can define this class of errors as follows.

\section{Definition 3 (Change interaction error (CIE))}

A change interaction error occurs if there exists a sequence $\vec{C}$ consisting of at least two changed statements, such that: $1)$ there exists an input $i$ that exercises all changed statements in $\vec{C}$ in order and $P(i) \not \equiv P^{\prime}(i) \wedge S(i) \not \equiv P^{\prime}(i)$; and 2) for every input $j$ that skips the execution of at least one changed statement in $\vec{C}$, we have that $S(j) \equiv P^{\prime}(j)$.

We call the sequence $\vec{C}$ a critical sequence of the CIE because it corresponds to a sequence of changed statements that needs to be exercised in the given order to expose the differential error. Intuitively, if every input exercising smaller sequences than $\vec{C}$ does not expose an error but some input exercising $\vec{C}$ in order exposes a differential error, then this error is due to the interaction among the statements $c \in \vec{C}$.

\subsection{Running Example}

For illustration purposes, we use the two concrete program versions $P$ and $P^{\prime}$ in Figure 5 to explain salient concepts in the remainder of this work. The two programs are simplified extracts of two versions of the Linux core utility cut - the behavior of which is explained in Section 2.2.1. The code is related to the parsing of the user-provided number ranges for the tool. As long as $* f$ s points to a character of the string, it tests whether the character is a digit (line 1), a dash (line 6) or the end of the line (line 9). If the character is a digit, then the number is read into value $\mathrm{v}$. In the changed version a boolean $\mathrm{lhs}$ is set to true (lines 4-5). If the character is a dash, the variable init is computed using $\mathrm{v}$ (lines 7-8). If the end of the line is reached, the bug is observable if init is 0 (line 10). 

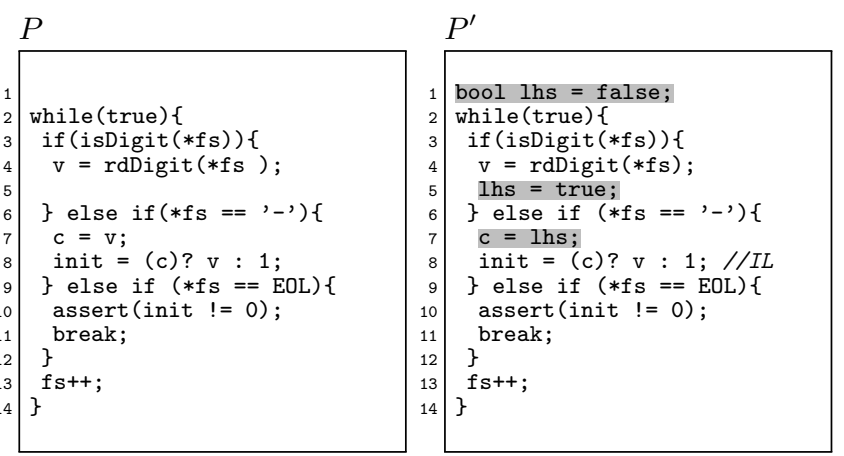

Figure 5: Core Utility cut.v1 changed to cut.v2

The changed statements are highlighted in grey. There are three changed statements in the changed version, which can be identified using the corresponding line numbers: $\{1,5,7\}$. We should point out that our notion of change is syntactic, purely textual and corresponds to code changes that manifest in the changed version $\left(P^{\prime}\right)$, such as added or modified statements. In other words, changed statements can be determined using textual differencing tools, like diff. The use of diff has the advantage that it works for any two programs, although it can be quite imprecise. There are other, more precise ways to deal with changes, but these typically assume some form of alignment between the two program versions [1, 13]. Unfortunately, these alignment assumptions do not always hold for the real programs that we are interested in. For this reason we chose the less precise, but unrestricted approach using diff.

Change Interaction Error. In the program $P^{\prime}$ in Figure 5 a CIE happens when the input string is " $0-"$ ". In this case the following sequence of changed statements is executed: $\langle 1,5,7\rangle$. Before entering the loop, line 1 is executed. Since the first character is ' 0 ', the first iteration of the loop meets the condition at line 3 and the changed statement in line 5 is executed. At this point the variable $v$ is set to 0 and the variable 1 hs is set to true. In the second iteration of the loop the condition at line 6 is met and the change in line 7 is executed. Since $\mathrm{lhs}$ is true, init is set to 0 (as $\mathrm{v}$ is 0 ). In contrast, for the same input, program $P$ sets the variable init to 1. Consequently, in the last iteration of the loop, the assertion in line 10 is violated for $P^{\prime}$, but not for $P$.

Note that only a specific sequence of changes (as well as a specific input) triggers this error. The interaction of the changed statements in lines 5 and 7 at the statement in line 8 causes this error. The combined semantic impact of both changes lead to the differential evaluation of the conditional expression (c)?v: 1 in both versions, $P$ and $P^{\prime}$. Other change sequences, such as $\langle 1,7,7\rangle,\langle 1,7,5\rangle,\langle 1,7,7,5\rangle$, will not expose the error.

\section{CHANGE SEQUENCE GRAPH}

To support detection of change interaction errors (CIE) we propose a statically computed structure which we call change sequence graph (CSG). A change sequence graph approximates the computation of potential CIEs by using control-flow information to derive sequences in which the changed statements can potentially be exercised and dependence information to derive locations at which the changes can potentially interact.

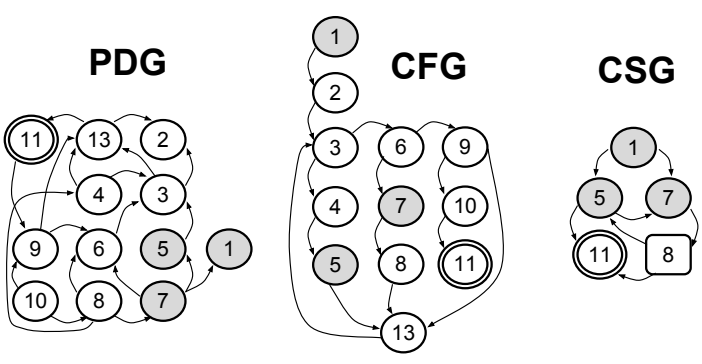

Figure 6: PDG, CFG, and CSG for $P^{\prime}$ in Figure 5.

\subsection{Potential Interaction}

To aid detecting CIEs, we can approximate all potential sequences of changed statements in a program using control-flow information from a control-flow graph (CFG). Essentially, a potential sequence of changed statements corresponds to a path in the CFG that contains changed statements. Having information about every potential sequences of changed statements is helpful because all critical sequences will be included in those sequences. In other words, this information will allow us to build a detection method for CIEs that searches potential critical sequences and exposes CIEs.

We are particularly interested in change sequences where the changed statements interact. That is, each executed changed statement has some impact on the output, and not executing one of those statements can lead to a different output. It is in this class of sequences where we can find change interaction errors. To detect such sequences, one useful definition is that of a potential interaction location of a sequence of changed statements.

\section{Definition 4 (Potential Interaction Location)}

A statement $s$ is a potential interaction location of a set of changed statements $C$, if $s$ (statically) data- or controldepends on every $c \in C$.

Information about potential interaction points can be computed using the program dependency graph (PDG). Essentially, we utilize the backward slice of the statement $s$ to compute the set of changed statements that can have a semantic impact on $s$. If the set contains more than one different changed statement, then $s$ is a potential interaction location of those statements. Note that an interaction location can coincide with a changed statement.

In Figure 6, the graphs on the left and in the middle depict the PDG and the CFG for our running example, respectively. The statement at line 8 is a potential interaction location of the changed statements in lines 5 and 7 , since it transitively depends on both the changed statements. As such, both changes can have a combined semantic impact on this control-location, effectively causing the regression error.

The notion of potential interaction location allows us to define an approximation of change interaction.

\section{Definition 5 (Potential Change Interaction)}

A set of changed statements $\vec{C}$ is potentially interacting if there are potential interaction locations for $\vec{C}$.

If there is no interaction location for two changed statements, since there is no information-flow, they are guaranteed not to interact and can be tested in isolation. 


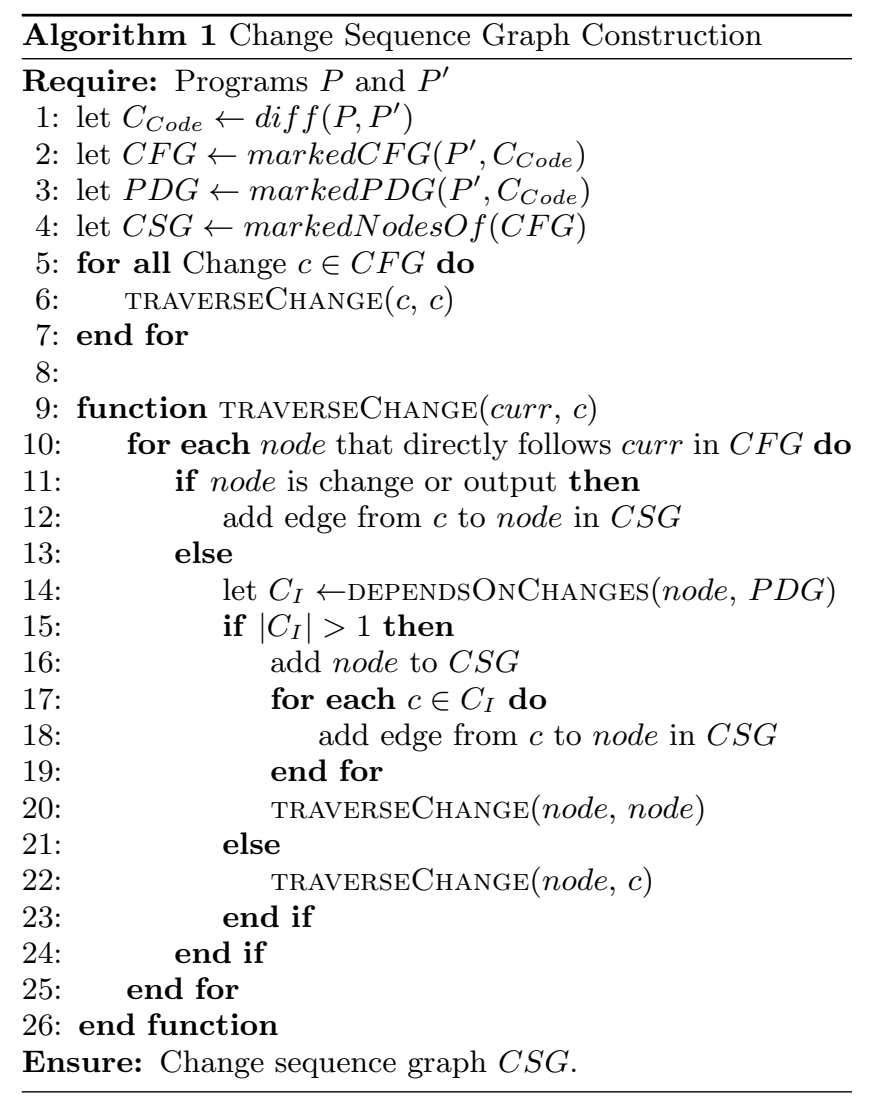

The information about all potential sequences of changed statements and potential interaction points can be synthesized in a change sequence graph (CSG). Thus a CSG represented a subset of program paths in a program where change interaction errors may exist. Other program paths, which are not represented in the CSG, cannot have change interaction errors as they do not contain change sequences.

\subsection{Computing the Change Sequence Graph}

The CSG can be computed using the CFG and the PDG for the changed program $P^{\prime}$. Algorithm 1 shows the detailed construction of the CSG. The inputs of the algorithm are two programs $P$ and $P^{\prime}$ and the output is the change sequence graph $C S G$. The first step is to compute the changed statements between $P$ and $P^{\prime}$ (line 1). As discussed in Section 3, this can be done using the diff tool. The next step is to compute the annotated versions of the CFG and PDG of $P^{\prime}$ (lines 2-3). Both, the CFG and PDG are annotated with information about the changed statements. Initially the CSG contains no edges, only nodes. These nodes are the changed statements and output nodes that are recovered from the CFG using the procedure markedNodesOf (line 4). The final step of the algorithm is to iterate through all the changed statements in the CFG and, for each change, use the auxiliary function TRAVERSECHANGE to add the relevant edges and interaction locations to the CSG (lines $5-7$ ).

The recursive function TRAVERSECHANGE takes two arguments curr and $c$. The first argument represents the current node in the CFG. The second argument represents the changed statement that edges may have to connect to. For each node in the CFG, which directly follows from the current node, we have three possibilities for the node:
Change or output node (lines $11-12$ ): If we reach some other change node, this indicates that there may be a control-flow from the change $c$ to this change. Thus, we add a corresponding edge to the CSG to indicate such potential flow. Similarly, if we reach an output node, we should add an edge between change $c$ and that node to indicate the potential control-flow.

Interaction location (lines $14-20$ ): If the node is an interaction location, it is added to the CSG and connected. Specifically, the function DEPENDsOnCHANGES(node, $P D G$ ) computes the changed statements that can have a semantic input on node using the $P D G$. If there is more than one change having a semantic impact on node, then node is an interaction location and is added to the CSG connected to the changes it depends on. Conceptually, every interaction location can be regarded as a new change. To trace the semantic impact of the interaction location, we keep recursively traversing the CFG by invoking TRAVERSECHANGE with both arguments set to node. Naively, the function DEPENDSONCHANGES can return all changed statements in the static backward slice of node. For optimization purposes, the function may choose a node to be an interaction location only if node is an "important" interaction location in some respect. For instance, given an interaction location $i$ for change sequence $\vec{C}$ and a statement $s$ that directly depends on $i$ - while $s$ is now also considered an interaction location of $\vec{C}$, it may not be an important one. Alternatively, the interaction locations could be computed by taking the static forward slice for every changed statement and marking their intersection as an interaction location.

Neither of above (line 22): Any other CFG node should be ignored in the CSG. This is achieved by calling TRAVERSECHANGE with node as first argument. This sets curr, representing the node in the CFG that is currently traversed, to node and implies that node will not appear in the CSG.

\section{SEARCH-BASED INPUT GENERATION}

To expose change interaction errors, and differential errors in general, test cases are generated. The exploration technique uses the Change Sequence Graph as a guide to exercises the structure of inter-dependencies across the changed statements. We employ symbolic execution along these dependencies.

The search-based input generation is depicted in Algorithm 2. The algorithm takes two program versions, $P$ and $P^{\prime}$, and the CSG (cf. Alg. 1) as input and computes a set of difference-revealing test cases $T$. We adopted the directed symbolic execution algorithm as discussed by Ma et al. [9] However, instead of searching for input that exercises any target in a specified (flat) set of targets, we extended the algorithm to search a specified directed graph of targets (i.e., the $C S G)$. The search algorithm is presented independent of the search strategy.

Algorithm 2 is initialized in the first five lines. It starts with an empty test suite $T$ and the first set of changed statements in the $C S G$ (those without incoming edges). These are added as targets for the symbolic state symbState which is created in lines 2-4. A symbolic state is essentially an intermediate state of symbolic execution and has three main properties - (i) a statement next which is to be executed next, (ii) a partial path condition $p c$, that is satisfied by every input exercising the same program path until $s$, and (iii) a set of targets. 


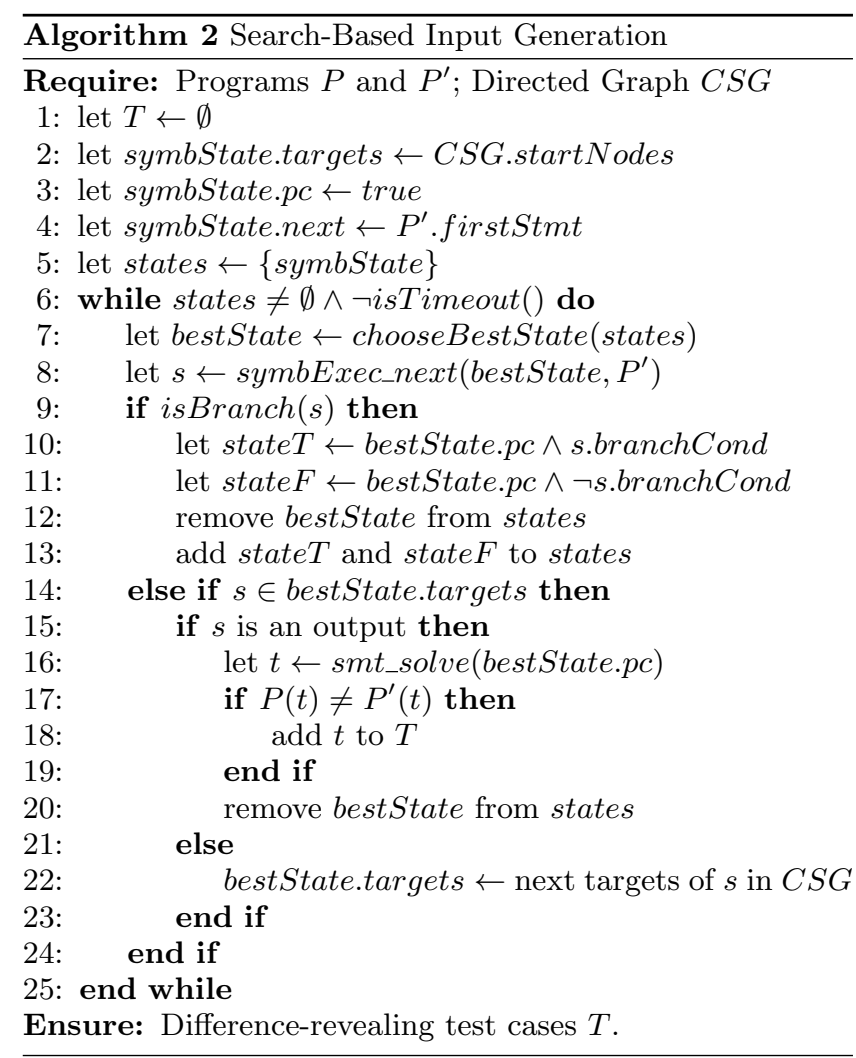

The symbolic execution of a symbolic state can be resumed at any time and pauses if a branch or a target is reached. The first symbolic state symbState is created with $p c=$ true, statement next is set program start, and the targets are assigned to the first set of changed statements in line 4 . In the following line 5 , it is added to the empty list of symbolic states.

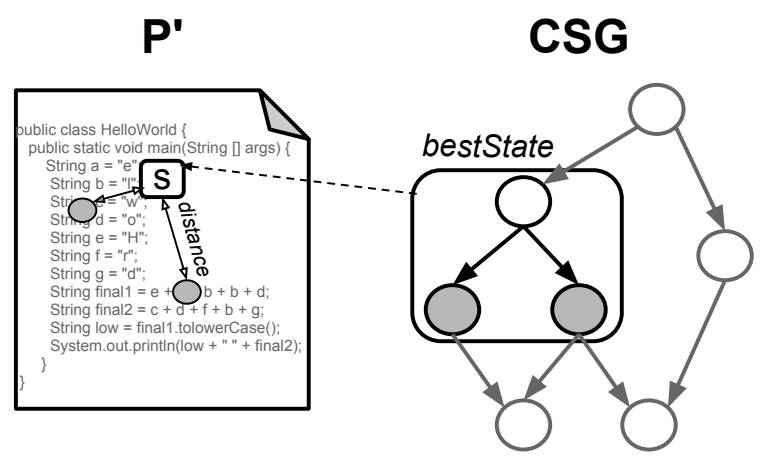

Figure 7: The bestState is chosen with the shortest distance in the source code of $P^{\prime}$ from $s$ to the target (left). Once a target is reached, the symbolic state moves to the target's children in the CSG (right).

The search commences in line 6. As long as the list of states is non-empty and no timeout occurs, the search works as follows. From the list of states the bestState is chosen according to a given search strategy, which is implemented in chooseBestState. For instance, as depicted in Figure 7, every symbolic state is assigned a measure of distance to its targets, ranked according to this measure, and chosen if it has the shortest distance. We further prioritize states with a greater proportion of targets that are yet unreached by other symbolic states. In line 8 , the bestState resumes the symbolic execution of $P^{\prime}$ until $s$, the next statement to be executed, becomes either a branch or one of the targets to be reached. If $s$ is a branch (lines 9-12), then two states are created - one following the true-branch and the other following the false-branch. The path conditions and the list of states are updated accordingly. If $s$ is a target (lines 1323 ), then we further distinguish whether or not $s$ is an output statement. If $s$ is an output statement, then we solve the path condition using a Satisfiability Modulo Theory solver to derive a concrete program input $t$ (line 15). This input is executed on both versions to validate whether $t$ exposes a behavioral difference. If so, $t$ is added to the set of differencerevealing test cases $T$. Since bestState reached the output, it requires no further symbolic execution and can be removed from the list of states (line 19). Otherwise, if $s$ is a target of bestState and not an output statement (line 21), then we set as new targets of bestState the nodes following the outgoing edges of the reached node in the $C S G$. The right-hand side of Figure 7 shows the bestState searching for two CSG nodes (in grey). If bestState finds the node on the left, the next target of bestState becomes that bottom left node.

\section{EMPIRICAL EVALUATION}

\subsection{Implementation and Setup}

We have implemented Algorithm 2 into the directed symbolic execution tool, Otter [9]. The user provides two versions of a $\mathrm{C}$ program compiled into the $\mathrm{C}$ Intermediate Language (CIL) and a text file with a representation of the CSG.

Otter provides a wide choice of search strategies which implement the function chooseBestState in Algorithm 2. For our experiments we used one of the most efficient ${ }^{8}$ strategies. The best symbolic state is chosen based on the shortest distance to the targets computed in the interprocedural control-flow graph. Occasionally, the next state is chosen randomly. We extended the search strategy by prioritizing states with a greater proportion of yet unreached targets. Instead of searching for a global set of targets, our implementation extends a symbolic state to have its own set of targets. Once a target is reached, the children of the reached target become the new targets for this state. The execution of a symbolic state terminates only if the output has been reached and thus no more further targets are to be reached. We then compute a concrete input satisfying the path condition of a state that reached an output node. This input is executed on both program versions. The information from the standard unix pipes stdout and stderr describes the program output. If the output differs in more than the program name, the test case and its differing output is reported. If the user optionally provides a "golden version", our implementation can classify the observed differential output further as "differential error" (i.e., regression/incomplete fix) or "progression".

We executed our implementation on a desktop computer with an Intel $\AA$ Core ${ }^{\mathrm{TM}_{2}}$ Quad CPU at $2.83 \mathrm{GHz}$ and $4 \mathrm{~GB}$ of main memory to generate test cases within the time frame of 5 minutes. The same sequence of changed statements can be exercised by multiple generated test cases.

\footnotetext{
${ }^{8}$ RoundRobin (RandomPath, InterSDSE-efficient).
} 


\subsection{Subjects}

We chose the subjects according to the following criteria:

Known Regressions. For every regression, we know i) the earlier version, ii) the regression-introducing version, iii) the bug report(s), and iv) the regressing-fixing version(s). The analysis of known regressions increases the credibility of the subjects and reduces the scope of non-maintenance commits which we need to inspect.

Multiple Changes. In this study, we are not interested in the semantic impact of single changed statements but the interplay of multiple changed statements. Therefore, we consider only regressions involving multiple changed statements.

Deterministic Behavior. The execution of the same input on the same program always yields the same output. Determinism is a prerequesite for every testing technique and as such also for symbolic execution.

\begin{tabular}{|c|c|c|c|}
\hline Version Pair & $\begin{array}{l}\text { Fixed in } \\
\text { Revision }\end{array}$ & $\begin{array}{l}\text { Commit } \\
\text { Date }\end{array}$ & $\begin{array}{c}\text { Bug Report [5] @ } \\
\text { http://lists.gnu.org/ }\end{array}$ \\
\hline seq.v0 $\rightarrow$ seq.v1 & seq.v2 & 09.07 .2007 & $2007-07 / \mathrm{msg} 00055 . \mathrm{html}$ \\
\hline $16.06 .05 \rightarrow 01.07 .06$ & seq.v3 & 14.02 .2009 & 2009-02/msg00139. html \\
\hline & seq.v3 & 14.02 .2009 & 2009-02/msg00139.html \\
\hline seq.v1 $\rightarrow$ seq.v2 & seq.v4 & 24.11 .2012 & 2012-11/msg00145.html \\
\hline $01.07 .06 \rightarrow 09.07 .07$ & seq.v5 & 10.01 .2013 & 2013-01/msg00054.html \\
\hline cut.v0 $\rightarrow$ cut.v1 & cut.v4 & 07.02 .2011 & $2011-02 / \mathrm{msg} 00036 . \mathrm{html}$ \\
\hline $02.06 .04 \rightarrow 04.12 .04$ & cut.v6 & 24.11 .2012 & 2012-11/msg00151.html \\
\hline cut.v1 $\rightarrow$ cut.v2 & cut.v3 & 22.05 .2007 & 2007-05/msg00195.html \\
\hline $04.12 .04 \rightarrow 22.05 .07$ & cut.v5 & 18.11.2012 & 2012-11/msg00114.html \\
\hline $\begin{array}{c}\text { cut.v6 } \rightarrow \text { cut.v7 } \\
24.11 .12 \rightarrow 06.12 .12\end{array}$ & cut.v8 & 05.02 .2013 & 2013-02/msg00011.html \\
\hline $\begin{array}{c}\text { expr.v0 } \rightarrow \text { expr.v1 } \\
16.11 .04 \rightarrow 14.01 .05\end{array}$ & expr.v2 & 26.05 .2005 & 2005-05/msg00189.html \\
\hline
\end{tabular}

Table 1: Six version pairs introducing eleven errors.

We study six version pairs that together introduced 11 differential errors, five of which are found and reported by our method (in bold font). Table 1 shows the considered Version Pairs the latter version of which introduces errors that are Fixed in the a subsequent revision. The fixes are presented with Commit Date and Bug Report. The bug being fixed with cut.v4 is further discussed by Marinescu and Cadar [10] and together with cut.v8 only observable as buffer overflow. We inserted an assertion that states that an array shall never be accessed out of bounds. The tools cut, seq, and expr consist of about 900, 500, and 900 Lines of Code (LoC), respectively. However, these tools utilize monolithic, shared libraries, prompting colleagues to quote between $2 \mathrm{k}$ to $3 \mathrm{k}$ effective LoC for the smallest tools [3] up to $20 \mathrm{k}$ instructions for the largest tool [10] in GNU Coreutils.

\subsection{Research Questions}

During the empirical evaluation of the change interaction guided regression test generation technique, we want to answer the following research questions.

RQ.1 Severity. How many differential errors can be classified as change interaction errors? What is the probability to exercise a sequence exposing a change interaction error compared to other sequences?

RQ.2 Efficacy. How many differential errors are exposed by a test generation technique that exercises changes in isolation as compared to one that considers their inter-dependencies and change interactions?

\section{RESULTS AND ANALYSIS}

\subsection{Result Presentation}

Table 2 shows the errors introduced when changing the given versions, whether these are change interaction errors and the test cases generated by our CSG-guided test generation technique. The first two columns show the errors introduced by the changes of the Version Pairs that are Fixed in the versions given in the second column. For instance, when seq.v1 was changed to seq.v2, errors are introduced that are fixed in versions seq.v3, seq.v4, and seq.v5. Errors fixed in revisions highlighted in bold face were previously unknown and subsequently reported by us. The following four columns show the results for the generation of test cases exercising the Change Sequence Graph, while the latter three columns show the results for a test generation technique that considers sufficient to exercise every changed statement, effectively treating Changes in Isolation. Both groups of columns have a similar format. Column \#Tests depicts the number of test cases generated. Column \#Diff depicts the number of test cases revealing a difference when executed on both versions. Some of the semantic differences are expected (progression). Column \#Error depicts the number of test cases exposing the given error. As per Definition 3, a differential error, that is exposed only by input exercising a sequence of changed statements but not by input "skipping" statements in that sequence, is classified as change interaction error (Col. CIE).

\begin{tabular}{|c|l|r|r|}
\hline Version Pairs & Sequence & \%Test & \%Error \\
\hline \multirow{2}{*}{ seq.v0 $\rightarrow$ seq.v1 } & non-critical & $19.02 \%$ & $0.00 \%$ \\
& critical & $80.98 \%$ & $1.39 \%$ \\
\hline \multirow{2}{*}{ seq.v1 $\rightarrow$ seq.v2 } & non-critical & $99.50 \%$ & $0.30 \%$ \\
& critical & $0.50 \%$ & $100.00 \%$ \\
\hline \multirow{2}{*}{ cut.v0 $\rightarrow$ cut.v1 } & non-critical & $96.83 \%$ & $4.09 \%$ \\
& critical & $3.17 \%$ & $100.00 \%$ \\
\hline \multirow{2}{*}{ cut.v1 $\rightarrow$ cut.v2 } & non-critical & $87.40 \%$ & $11.71 \%$ \\
& critical & $12.60 \%$ & $33.33 \%$ \\
\hline \multirow{2}{*}{ cut.v6 $\rightarrow$ cut.v7 } & non-critical & $95.68 \%$ & $0.00 \%$ \\
& critical & $4.32 \%$ & $28.57 \%$ \\
\hline \multirow{2}{*}{ expr.v0 $\rightarrow$ expr.v1 } & non-critical & $71.43 \%$ & $0.00 \%$ \\
& critical & $28.57 \%$ & $16.67 \%$ \\
\hline
\end{tabular}

Table 3: \%Test of generated tests exercise a (non-) critical sequence. \%Error of generated tests, that exercise a (non-) critical sequence, expose an error.

Table 3 shows the percentage of tests exercising critical sequences versus the percentage of tests exercising non-critical sequences. One test case exercises exactly one sequence. A critical sequence is a sequence of changed statements that is relevant to expose a change interaction error. The first column depicts the Version pairs considered, followed by whether the results refer to critical or non-critical sequences. The latter two columns are explained by example of the last row: "On average, one quarter of the generated test cases for the version pair expr.v0 and expr.v1 exercise a critical sequence. From those, every sixth exposes an error".

To generate the test suites that stress changes in isolation (see RQ.2), we generated test cases that cover every changed statement that is also exercised by the approach presented in this paper. We set as targets the output and such statements that have the greatest depth in the chain of control-dependencies. In other words, instead of a graph of targets, we provided a set of targets. Otherwise, we employed the same tool, search strategy, and time frame. 


\begin{tabular}{|c|c|c|c|c|c|c|c|c|}
\hline \multirow[b]{2}{*}{ Version Pair } & \multirow[b]{2}{*}{ Fixed in } & \multicolumn{4}{|c|}{ RQ1: Change Sequence Graph } & \multicolumn{3}{|c|}{ RQ2: Changes in Isolation } \\
\hline & & CIE & \#Tests & \#Diff & \#Error & \#Tests & \#Diff & \#Error \\
\hline \multirow{2}{*}{ seq.v0 $\rightarrow$ seq.v1 } & seq.v2 & $\mathrm{X}$ & 16163 & 43 & 6 & 205 & 65 & 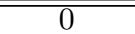 \\
\hline & seq.v3 & $\mathrm{x}$ & 163 & 43 & 5 & 205 & 65 & 0 \\
\hline \multirow{3}{*}{ seq.v1 $\rightarrow$ seq.v2 } & seq.v3 & - & 200 & 26 & 2 & 200 & 21 & $\overline{17}$ \\
\hline & seq.v4 & - & 200 & 26 & 3 & 200 & 21 & 0 \\
\hline & seq.v5 & $\mathrm{x}$ & 200 & 26 & 1 & 200 & 21 & 0 \\
\hline \multirow{2}{*}{ cut.v0 $\rightarrow$ cut.v 1} & cut.v4 & 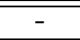 & 379 & 42 & 30 & 471 & 42 & 30 \\
\hline & cut.v6 & $\mathrm{x}$ & 379 & 42 & 12 & 471 & 42 & 12 \\
\hline \multirow{2}{*}{ cut.v1 $\rightarrow$ cut.v2 } & cut.v3 & - & 254 & 228 & 162 & 453 & 201 & 58 \\
\hline & cut.v5 & $\mathrm{x}$ & 254 & 228 & 26 & 453 & 201 & 5 \\
\hline cut.v6 $\rightarrow$ cut.v7 & cut.v8 & $\overline{\mathrm{X}}$ & 324 & 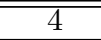 & 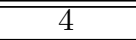 & 342 & 6 & $\overline{6}$ \\
\hline expr.v0 $\rightarrow$ expr.v1 & expr.v2 & $\mathrm{x}$ & 42 & 2 & 2 & 82 & 2 & 2 \\
\hline \multicolumn{2}{|c|}{ Average (per version pair) } & $7 / 11$ & 227 & 57.5 & 46.3 & 292.2 & $\overline{55.8}$ & 21.7 \\
\hline
\end{tabular}

Table 2: Errors introduced, fixed in later versions, are witnessed by test cases generated within 5 minutes.

\section{RQ.1 Change Interaction Errors}

Two third of the analyzed differential errors are change interaction errors. Only one in five test cases exercise a critical sequence, being 15 times more likely to expose an error.

Using our implementation, we have found and reported four of the seven listed change interaction errors and one more differential error, that were previously unknown. On average, 227 test cases were generated that exercise a change sequence (see Table 2). Every fourth test case propagates the combined semantic effect of the exercised changed statements to the output and thus makes a difference observable. While many of these expose expected behavioral changes, every fifth test case exposes a differential error.

Change interaction errors are subtle. On average, only $21.7 \%$ of the generated test cases exercise a critical sequence (see Table 3). On the other hand, the malicious effect of a critical change sequence is much greater than that of a noncritical sequence. Only $3.2 \%$ of the test cases exercising a non-critical sequence expose an error versus $50 \%$ exercising a critical sequence. Test cases exercising a critical sequence are 15.6 times more likely to expose an error than test cases exercising a non-critical sequence. That suggests that the changes in these critical sequences are interacting in a negative and unintended manner.

\section{RQ.2 Comparison to Changes in Isolation}

Only $57 \%$ of the change interaction errors are exposed by test cases generated to stress changes in isolation.

To compare, we generated a test suite that covers every changed statement which is also covered by the test suite generated using a change sequence graph. On average, 292 test cases were generated that exercise a change sequence (cf. Table 2). Every fifth test case propagates the combined semantic effect of the exercised changed statements to the output and thus makes a difference observable. Many of these expose expected behavioral changes, every 15th test case exposes a differential error - significantly less than our CSG-based test generation approach. Within five minutes, using our CSG-based approach every error is witnessed by 25 test cases on average. In contrast, using the other approach that considers changes in isolation only seven of the eleven errors are witnessed by, on average, 18 test cases each. In particular, only $57 \%$ of the change interaction errors are exposed by test cases generated to stress changes in isolation as compared to $100 \%$ by our technique.

\section{THREATS TO VALIDITY}

The main threat to external validity is the generalization of the results. During our study of GNU Coreutils we encountered several regression errors that can only be observed when certain environmental conditions are satisfied. One example is an error that was reported to occur specifically on a Solaris 32-bit machine and could not be reproduced on other machines. Depending on the program environment, the same test case may or may not expose an error. In fact, the package co-maintainer of GNU Coreutils, Pádraig Brady, noted in an email correspondence that it may be unclear even for the experienced developer, exactly how to write the test cases in the presence of such non-determinism. He suggested to introduce an explicit interface for file operations. This suggests a lack of modelling the environment [14], or concurrency [20] during the testing process. As discussed in Section 6.2, our experimental subjects and regression errors are chosen so that the observability of an error does not depend on the program environment but on source code properties. The conclusions should be viewed in the same context.

The main threats to internal validity are T.1) the search strategy that was utilized and T.2) the practical absence of assertions that mark an error within symbolic execution. T.1) The experimental results depend on the utilized search strategy. A less efficient search strategy may have exposed less differential errors within the same amount of time. However, the utilized search strategy does not prioritze critical over non-critical sequences. Thus, it does not affect the main conclusion of $R Q .1$. We utilized the same search strategy for the experiments that compares to testing changes in isolation. Thus, it does not affect the main conclusion of RQ.2. T.2) Symbolic execution requires highlighting of error states, for instance, by assertions. In Section 2 and Table 1, we list the versions cut.v4 and cut.v8 as bug fixes for regressions introduced in an earlier version of cut. The regressions are observable as buffer overflows. However, without the explicit assertion stating that an array should never be accessed at an index greater than its size, the symbolic index for this array may often concretize as small number, such as 1 or 0 , but never as a number that has more than the nine digits necessary to witness these particular overflows. While our implementation is able to find error-exposing test cases in the presence of such assertions, it is unable to find errorexposing test cases in their absence for such buffer-overflows. 


\section{RELATED WORK}

Test Suite Augmentation aims at generating new test cases that stress the changed behaviour in a program. Typically, this is done by exploiting knowledge about changes and using symbolic execution techniques - which are also key ideas in our approach. However, the main novelty of our work is the consideration of the inter-dependencies among multiple changes during test generation. Our technique effectively exercises sequences of changed statements and potential interaction locations. Existing techniques either discuss the semantic impact of single changes only [1, 13], or do not systematically consider the interaction and interdependencies among multiple code changes $[15,18]$.

Test Suite Augmentation techniques can be distinguished in semantic approaches $[2,11]$, that are based on the program summaries of both versions to compute the semantic changes, and syntactic approaches, that are directed by the syntactic changes to exercise paths that may expose semantic changes. The syntactic techniques can be further distinguished into those seeking to re-establish code coverage of a test suite after the program is changed [22], those following the Reach-Infect-Propagate ${ }^{9}$ approach [1, 15, 13], and those exercising every program path affected by a change $[18,12]$.

Techniques, such as eXpress [18] or DiSE [12], that exercise every program path affected by changed statements, are finer-grained and less scalable than our approach. The focus on affected code regions makes these techniques more efficient than full path exploration approaches, like DART [6], since less paths are to be explored. However, these techniques may still exercise many different paths within the same sequence of changed statements; paths that may or may not contain interaction locations; paths that may all expose the same error. More systematically, our CSG directed TSA approach targets sequences and interaction locations of changed statements instead of all affected paths. In practice, this means that once a difference revealing test case is found for a sequence, unexplored affected paths that can still realize this sequence do not no have to be explored further.

TSA techniques based on Reach-Infect-Propagate (RIP) $[15,13,16]$ follow a motivation similar to our work: Instead of exploring every path affected by changes, the RIP approaches deem it sufficient to find one path that executes a change, infects the program state, and propagates to the output. However, existing techniques consider the semantic effects of the changes in isolation. For the subjects in our experiments, a technique based on this consideration could expose only half of the change interaction errors. In the presence of multiple changes, the approach of Santelices et al. [15] requires a change-free path from the change to the program start - effectively a change in isolation.

Coverage-based TSA techniques seek to re-establish code coverage when the program is changed [22, 21]. However, to expose change-interaction errors and understand the combined semantic impact of multiple changes, it is insufficient to merely exercise every change, as discussed earlier.

Semantic TSA techniques $[2,11]$ require the computation of a differential semantic program summary for both versions to determine the semantic changes. While this approach is sound and very precise, it may be less scalable.

\footnotetext{
${ }^{9}$ Reach a change, infect the program state, and propagate the infection to the output [19].
}

Change Interaction. Santelices et al. [17] propose a formal definition of change interaction: two changes $c_{1}$ and $c_{2}$ interact in an execution if removing one of the changes alters the semantic effect of the other change on that execution. This notion of change interaction is too precise. For our practical purposes, detecting such changes interactions cannot be done in an efficient manner. Essentially, given a test case $t$ and code changes $C$ that are applied to program $P$ yielding $P^{\prime}$, there are $2^{|C|}$ program configurations to be analyzed, each with only a subset of $C$ applied to $P$. Our definition of potential change interaction approximates the above definition and can be computed more efficiently. A set of changed statements $C$ potentially interacts if there exists a statement that syntactically depends on every $c \in C$.

Reachability. In order to explore change sequences our approach builds and extends previous work that deals with reaching statements in a program. However, these tools seek to reach a single statement [13, 23], a set of statements [9], or a sequence of statements [8] instead of a graph. To overcome this problem we have modified the Otter tool [9] to take a graph of statements as input and target multiple statements along this graph structure at once.

\section{CONCLUSION}

When a program evolves due to feature additions, bug fixes, or other code quality improvements, the source code of the program is changed. Especially, when multiple developers change the code base at different places, comprehending the semantic impact of such code changes and potential interactions is difficult. Consequently, errors that result from a poor understanding of the inter-dependencies across changes are often introduced in the code base.

In this paper, we have argued for the importance and subtleness of such change interaction errors, which are pervasive even in well-tested and widely used software. Since existing regression test generation techniques do not adequately stress code where change interaction may occur, we have proposed a new regression test generation technique that addresses these limitations. Our recipe for exposing change-interaction errors employs a judicious mix of flows, dependencies and semantic effects across changes. In other words, to witness a change interaction error - multiple changes should be executed (flow information), multiple changes should affect a potential interaction location via data- and control dependencies (dependence information), and the semantic effect of a change should not get masked. In our approach, the control flow between changes is captured in the Change Sequence Graph, dependencies across changes are witnessed in potential interaction locations, and we attempt to exercise these dependencies and propagate their semantic effects via symbolic execution on the changed program. Our experiments on GNU Coreutils demonstrate the effectiveness of this approach in hunting down hard-tofind change-interaction errors even in well-tested software.

\section{ACKNOWLEDGMENTS}

We are grateful for the engaging discussions with the package maintainers of the GNU Coreutils, Jim Meyering and Pádraig Brady. Both shared their valueable insights in the testing of a real, widely distributed project that is being successfully maintained for over 20 years. This work was partially supported by Singapore's Ministry of Education research grant MOE2010-T2-2-073. 


\section{REFERENCES}

[1] T. Apiwattanapong, R. Santelices, P. K. Chittimalli, A. Orso, and M. J. Harrold. Matrix: Maintenance-oriented testing requirements identifier and examiner. In Proceedings of the Testing: Academic 86 Industrial Conference on Practice And Research Techniques, TAIC-PART '06, 2006.

[2] M. Böhme, B. C. Oliveira, and A. Roychoudhury. Partition-based regression verification. In Proceedings of the 2013 International Conference on Software Engineering, ICSE 2013, pages 301-310, 2013.

[3] C. Cadar, D. Dunbar, and D. Engler. Klee: unassisted and automatic generation of high-coverage tests for complex systems programs. In Proceedings of the 8th USENIX conference on Operating systems design and implementation, OSDI'08, pages 209-224, Berkeley, CA, USA, 2008. USENIX Association.

[4] C. Cowan, C. Pu, D. Maier, H. Hintony, J. Walpole, P. Bakke, S. Beattie, A. Grier, P. Wagle, and Q. Zhang. Stackguard: automatic adaptive detection and prevention of buffer-overflow attacks. In Proceedings of the 7th conference on USENIX Security Symposium - Volume 7, SSYM'98, pages 5-5, Berkeley, CA, USA, 1998. USENIX Association.

[5] GNU Coreutils - bug reports. http://lists.gnu.org/ archive/html/bug-coreutils/, Accessed: July 2013.

[6] P. Godefroid, N. Klarlund, and K. Sen. Dart: directed automated random testing. In Proceedings of the 2005 ACM SIGPLAN conference on Programming language design and implementation, PLDI '05, 2005.

[7] Z. Gu, E. T. Barr, D. J. Hamilton, and Z. Su. Has the bug really been fixed? In Proceedings of the 32nd ACM/IEEE International Conference on Software Engineering - Volume 1, ICSE '10, pages 55-64, New York, NY, USA, 2010. ACM.

[8] W. Jin and A. Orso. Bugredux: reproducing field failures for in-house debugging. In Proceedings of the 2012 International Conference on Software Engineering, ICSE 2012, 2012.

[9] K.-K. Ma, K. Y. Phang, J. S. Foster, and M. Hicks. Directed symbolic execution. In Proceedings of the 18th International Conference on Static Analysis, SAS'11, 2011.

[10] P. D. Marinescu and C. Cadar. make test-zesti: a symbolic execution solution for improving regression testing. In Proceedings of the 2012 International Conference on Software Engineering, ICSE 2012, pages 716-726, Piscataway, NJ, USA, 2012. IEEE Press.

[11] S. Person, M. B. Dwyer, S. Elbaum, and C. S. Păsăreanu. Differential symbolic execution. In Proceedings of the 16th ACM SIGSOFT International Symposium on Foundations of software engineering, SIGSOFT '08/FSE-16, pages 226-237, New York, NY, USA, 2008. ACM.

[12] S. Person, G. Yang, N. Rungta, and S. Khurshid.
Directed incremental symbolic execution. In Proceedings of the 32nd ACM SIGPLAN conference on Programming language design and implementation, PLDI '11, 2011.

[13] D. Qi, A. Roychoudhury, and Z. Liang. Test generation to expose changes in evolving programs. In Proceedings of the IEEE/ACM international conference on Automated software engineering, ASE '10, 2010.

[14] D. Qi, W. N. Sumner, F. Qin, M. Zheng, X. Zhang, and A. Roychoudhury. Modeling software execution environment. Reverse Engineering, Working Conference on, 0:415-424, 2012.

[15] R. Santelices, P. K. Chittimalli, T. Apiwattanapong, A. Orso, and M. J. Harrold. Test-suite augmentation for evolving software. In Proceedings of the 2008 23rd IEEE/ACM International Conference on Automated Software Engineering, ASE '08, 2008.

[16] R. Santelices and M. J. Harrold. Applying aggressive propagation-based strategies for testing changes. In Proceedings of the 2011 Fourth IEEE International Conference on Software Testing, Verification and Validation, ICST '11, pages 11-20, Washington, DC, USA, 2011. IEEE Computer Society.

[17] R. Santelices, M. J. Harrold, and A. Orso. Precisely detecting runtime change interactions for evolving software. In Proceedings of the 2010 Third International Conference on Software Testing, Verification and Validation, ICST '10, 2010.

[18] K. Taneja, T. Xie, N. Tillmann, and J. de Halleux. express: guided path exploration for efficient regression test generation. In Proceedings of the 2011 International Symposium on Software Testing and Analysis, ISSTA '11, 2011.

[19] J. M. Voas. Pie: A dynamic failure-based technique. IEEE Trans. Softw. Eng., 18(8):717-727, Aug. 1992.

[20] C. Wang, M. Said, and A. Gupta. Coverage guided systematic concurrency testing. In Proceedings of the 33rd International Conference on Software Engineering, ICSE '11, pages 221-230, New York, NY, USA, 2011. ACM.

[21] Z. Xu. Directed test suite augmentation. In Proceedings of the 33rd International Conference on Software Engineering, ICSE '11, pages 1110-1113, New York, NY, USA, 2011. ACM.

[22] Z. Xu, Y. Kim, M. Kim, G. Rothermel, and M. B. Cohen. Directed test suite augmentation: techniques and tradeoffs. In Proceedings of the eighteenth ACM SIGSOFT international symposium on Foundations of software engineering, FSE '10, pages 257-266, New York, NY, USA, 2010. ACM.

[23] C. Zamfir and G. Candea. Execution synthesis: a technique for automated software debugging. In Proceedings of the 5th European conference on Computer systems, EuroSys '10, 2010. 